\title{
A Matter of Controversy: Is Radioiodine Therapy Favorable in Differentiated Thyroid Carcinoma?
}

\author{
Matthias Schmidt ${ }^{1}$, Rainer Görges ${ }^{1}$, Alexander Drzezga ${ }^{2}$, and Markus Dietlein ${ }^{1}$ \\ ${ }^{1}$ University Hospital of Cologne, Cologne, Germany; and ${ }^{2}$ University Hospital of Essen, Essen, Germany
}

\begin{abstract}
Learning Objectives: On successful completion of this activity, participants should be able to describe (1) when radioiodine therapy was first introduced; (2) what special features of thyroid carcinoma distinguish it from other malignancies; and (3) which retrospective studies are needed to support the clinical usefulness of radioiodine therapy.
\end{abstract}

Financial Disclosure: Drs. Schmidt, Görges, Drzezga, and Dietlein received speaker fees from Sanofi GmbH. The authors of this article have indicated no other relevant relationships that could be perceived as a real or apparent conflict of interest.

CME Credit: SNMMI is accredited by the Accreditation Council for Continuing Medical Education (ACCME) to sponsor continuing education for physicians. SNMMI designates each JNM continuing education article for a maximum of 2.0 AMA PRA Category 1 Credits. Physicians should claim only credit commensurate with the extent of their participation in the activity. For CE credit, SAM, and other credit types, participants can access this activity through the SNMMI website (http://www.snmmilearningcenter.org) through August 2021.

See an invited perspective on this article on page 1184.

Radioiodine therapy is a matter of controversy because different opinions exist about its use for differentiated thyroid carcinoma. The following article sheds light on the different opinions and explains why we advocate the use of radioiodine therapy in more than only high-risk patients. In comparison to other malignancies, differentiated thyroid carcinoma has a different tumor biology due to its usually slow growth pattern. Radioiodine therapy was first used about 75 y ago and provided cure at a time when prospective randomized controlled trials had yet to be developed. Large patient cohorts and usually at least a decade of clinical follow-up are needed to demonstrate a benefit from radioiodine therapy. Thus, especially in low-risk patients, many factors define an individual treatment decision, including tumor stage, extent of surgery, tumor biology, clinical and imaging data, life expectancy, and patient preferences.

Key Words: differentiated thyroid carcinoma; papillary thyroid carcinoma; follicular thyroid carcinoma; radioiodine therapy

J Nucl Med 2018; 59:1195-1201

DOI: 10.2967/jnumed.117.191338

\section{A}

fter the discovery of ${ }^{131}$ I by Glenn Seaborg and John Livingood at the University of California, Berkley, in 1938, Saul Hertz administered the first therapeutic dose of cyclotron-produced ${ }^{131} \mathrm{I}$ to a human patient in January 1941 (1). To remember the founder of radioiodine therapy, the 2016 congress of the European Association of Nuclear Medicine in Barcelona held a symposium on Saul Hertz, in the presence of his daughter Barbara Hertz. Today, there is strong controversy over whether to use radioiodine in patients with differentiated thyroid carcinoma (DTC). The following article

Received Jan. 21, 2018; revision accepted May 3, 2018.

For correspondence or reprints contact: Matthias Schmidt, Department of Nuclear Medicine, University Hospital of Cologne, Kerpener Strasse 62, D-50937 Koeln (Cologne), Germany.

E-mail: matthias.schmidt@uk-koeln.de

Published online May 10, 2018.

COPYRIGHT (C 2018 by the Society of Nuclear Medicine and Molecular Imaging. presents the different views on radioiodine therapy and explains why we advocate the use of radioiodine.

\section{DTC HISTOPATHOLOGY, METASTATIC PATTERNS, AND TREATMENT AIMS}

DTC consists of two histologic types, papillary thyroid carcinoma (PTC) and follicular thyroid carcinoma (FTC). In general, both entities are slowly growing. Prognosis is good if completely eradicated, but the metastatic patterns of these two types of carcinoma differ. FTC metastasizes predominantly to distant organs such as lungs and bones, whereas PTC shows a high incidence of lymph node metastasis, ranging from $30 \%$ to $80 \%$ of patients $(2,3)$. Surgery has the goal of removing the tumor, and radioiodine therapy has two goals: ablation of thyroid remnants to facilitate follow-up and - more importantly-destruction of microscopic tumor tissue as an adjuvant therapy (4). DTC in pregnant women is, fortunately, rare, as pregnancy is an absolute contraindication to the use of radioiodine therapy.

\section{TOTAL THYROIDECTOMY VERSUS LOBECTOMY}

Thyroid lobectomy alone is also sufficient for minimally invasive FTC without angioinvasion $(5,6)$ and for encapsulated follicular-patterned thyroid tumors of uncertain malignant potential according to the 4th edition of the World Health Organization classification of tumors (e.g., noninvasive follicular thyroid neoplasm with papillarylike nuclear features [NIFTP]) (7). The presence of the remaining lobe of the gland does not allow for the use of radioiodine remnant ablation. Follow-up management consists of neck ultrasonography and serial thyroglobulin measurements. For patients with an unequivocal thyroid cancer larger than $1.0 \mathrm{~cm}$, we recommend a near-total or total thyroidectomy. Then, the question arises of whether radioiodine ablation may improve recurrence-free or overall survival.

\section{METHODOLOGIC ASPECTS OF RADIOIODINE THERAPY}

There are several reasons for controversy in the use of radioiodine. There is a lack of prospective randomized controlled trials, and data on clinical outcomes are taken mostly from retrospective series. To set up prospective randomized trials with radioiodine is not an easy task, requiring long-term follow-up because of the slowly growing tumor biology and the low event rate. In a review 
by Sawka et al., only studies with an observation period of more than $10 \mathrm{y}$ showed a benefit for patients treated with radioiodine, and sample sizes required recruitment of at least 1,500 patients $(8,9)$. There are ongoing prospective clinical trials aiming to further clarify the indication for radioiodine therapy, such as the French multicenter "Etude Stimulation Ablation" (ESTIMABL trial) (10), the British "Is Ablative Radio-Iodine Necessary for Low Risk Differentiated Thyroid Cancer Patients" (IoN trial) $(11,12)$, and the German "I-124 PET/CT Based Remnant Radioiodine Ablation Decision Concept in Differentiated Thyroid Cancer" (CLERAD-PROBE trial) (13), which include low-risk patients. Results are expected beginning in 2020. The difficulty in planning prospective studies can be exemplified by considering the feasibility of designing a multiinstitutional prospective randomized controlled trial of prophylactic central lymph node dissection in cN0 PTC. Carling et al. calculated that a total of 5,840 patients would have to be included in such a study to achieve at least $80 \%$ statistical power. Thus, such a study was considered not readily feasible (14).

\section{GUIDELINES OF AMERICAN THYROID ASSOCIATION (ATA) AND BRITISH THYROID ASSOCIATION}

In 2015, the ATA published version 3 of the guidelines dealing with the diagnosis and treatment of DTC (6). The recurrence risk was expanded from the basic 3-tiered system of low, intermediate, and high risk for locoregional recurrence or distant metastases to a broader risk continuum that incorporates actual risk percentages from several cited studies $(6,15)$. In version 3 of the ATA guidelines, patients with lymph node metastases can be categorized into each of the 3 risk groups rather than simply placing all patients with lymph node disease into the intermediate-risk category $(6,15)$. Only patients who have tumors with gross extrathyroidal invasion (T4) - together with $\mathrm{pN} 1$ with extranodal extension and at least 3 lymph nodes involved, any lymph node larger than $3 \mathrm{~cm}$, or distant metastases - are definite candidates for radioiodine therapy, whereas $\mathrm{T} 1 \mathrm{~b}$ and $\mathrm{T} 2$ tumors are not considered routine indications. Patients with other T categories such as T3 or T1-3 N1a or N1b are candidates in whom radioiodine therapy could be considered. The authors of the ATA guideline state that of the 191 individual recommendations, only 11 were based on highquality evidence whereas 97 were based on low-quality evidence (6). Haugen et al. aimed to reduce what they consider excess therapy and rather want to monitor patients with a few, small lymph node metastases who are now categorized in the low-risk category $(6,15)$. Noteworthy are considerations concerning remnant ablation, adjuvant therapy, and therapy of metastases. Patients who are considered for remnant ablation are treated with radioiodine to ablate normal thyroid tissue, and the ablation should improve the sensitivity and specificity of subsequent monitoring tools. Patients considered for adjuvant therapy are those who have undergone a complete resection of their disease and have no known residual disease but are at high risk of developing recurrent disease. Patients who receive radioiodine therapy are those who have known residual locoregional or metastatic disease and who need therapy in an attempt to eradicate or control the disease. Haugen et al. suggested that those patients who are considered for remnant ablation should be considered for only a lower administered activity of radioiodine (i.e., 1.1 $\mathrm{GBq}\left[30 \mathrm{mCi}\right.$ ] of ${ }^{131} \mathrm{I}$ ), reserving higher administered activities for those patients receiving radioiodine for adjuvant therapy or therapy of metastases. These approaches aim to reduce the risk of potential side effects (e.g., sialadenitis, xerostomia, epiphora, or secondary malignancy) in patients with low- or intermediate-risk DTC.

The British Thyroid Association guideline (4) gave similar recommendations on the use of radioiodine in DTC patients but used the term selective use instead of may be considered. Questions remain about the precise specification of selection criteria.

\section{POSITION OF EUROPEAN ASSOCIATION OF NUCLEAR MEDICINE AND EUROPEAN COUNTRIES}

In 2016, Verburg et al. published an article on why the European Association of Nuclear Medicine declined to endorse the 2015 ATA guidelines (16). In most cases, the objections were based on differences in the interpretation of the available evidence, especially where the role of nuclear medicine is concerned. The objections start with the acceptance of incomplete resection. The ATA recommendation either to perform a bilateral near-total or total thyroidectomy or to limit the initial procedure to lobectomy for tumors larger than $1 \mathrm{~cm}$ but smaller than $4 \mathrm{~cm}$, and with no extrathyroidal extension or clinical evidence of lymph node metastases ( $\mathrm{cNO}$ ), is a shift from previous clinical practice and would make adjuvant radioiodine therapy not feasible in the presence of a large thyroid remnant. This recommendation would result in a definite change in medical practice concerning state-of-the art follow-up examinations, because thyroglobulin would no longer have the significance it has today. For the entire population of patients with DTC exceeding $1 \mathrm{~cm}$ in diameter, the usefulness of postoperative ${ }^{131} \mathrm{I}$ ablation is evident from retrospective studies $(8,9)$. The expertise of the surgeon is an important parameter, and postoperative serum thyroglobulin may be an indicator of the completeness of thyroid tissue removal, helping to identify patients who may benefit from ${ }^{131}$ I therapy (16).

In Germany, it is recommended that patients be advised to undergo radioiodine therapy unless they have papillary thyroid microcarcinoma (PTMC) (17). Patients considered at low risk for recurrent disease (pT1b-pT2 cN0 M0; risk of recurrence, 2\%-7\%) are usually treated with radioiodine therapy. In our institution, 2.0 $\mathrm{GBq}$ of ${ }^{131} \mathrm{I}$ are usually administered in this situation. The ATA limited its own recommendation by judging it as weak, as based on low-quality evidence, and as lacking inclusion of more aggressive variants of DTC. The results of large retrospective studies in the United States are in contrast to the ATA recommendations.

\section{Low-Risk Patients}

The U.S. National Cancer Database from 1998 to 2006 indicates that radioiodine therapy significantly improved overall survival in 61,775 patients with PTC diameters of $1.0-2.0 \mathrm{~cm}$ and $2.1-4.0 \mathrm{~cm}(18)$. When average mortality was assigned a value of 1.0 , mortality decreased in the radioiodine therapy group to a value of 0.77 (95\% confidence interval $[\mathrm{CI}], 0.68-0.87$ ) in pT1b and to a value of 0.86 (95\% CI, 0.76-0.98) in pT2. The clinical benefit of radioiodine therapy versus no radioiodine therapy was higher than the clinical benefit of total thyroidectomy versus subtotal thyroidectomy. Total thyroidectomy did not improve overall survival in comparison to subtotal surgical procedures. These therapeutic results were not attributable to one fixed radioiodine activity but resulted from mean applied activities over 2 decades (Table 1) $(18,19)$.

The U.S. Surveillance, Epidemiology, and End Results (SEER) Database from 1973 to 2009 indicates that omission of radioiodine therapy increased disease-specific mortality in 61,049 patients. 


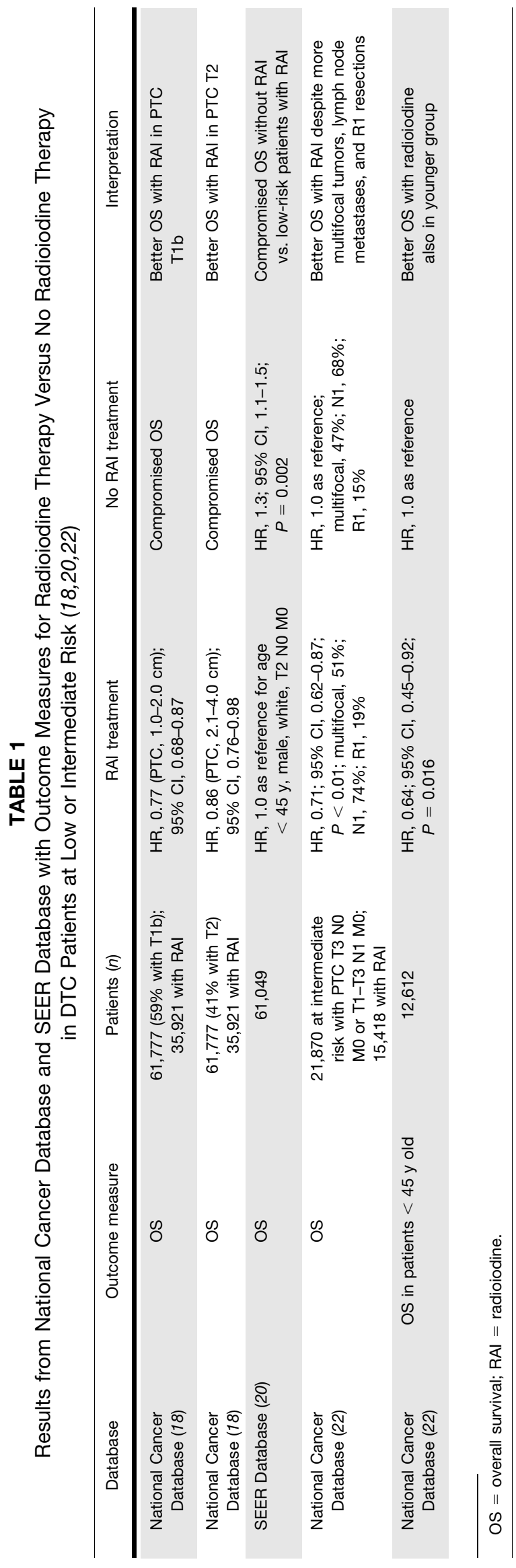

Male patients under 45 y old with pT2 N0 M0 disease had a significantly higher hazard ratio (HR), 1.3, if they did not receive radioiodine therapy $(P<0.002)$, while female sex showed a significant protective effect (HR, 0.7; $P=0.001$ ) (Table 1) (20). In a study from Hong Kong with 855 patients, recurrence-free survival increased from $82.5 \%$ to $95 \%$ after $10 \mathrm{y}$ when radioiodine was included in the therapeutic concept $(21)$. These data demonstrating a benefit for DTC patients were from retrospective series with very large patient collectives and a long follow-up.

\section{Intermediate-Risk Patients}

Adam et al. analyzed the prognostic value of lymph node metastases in a cohort of 47,902 patients from the SEER Database (19). Overall survival was compromised for patients with nodal metastases (HR, 1.32; 95\% CI, 1.04-1.67; $P<0.021$ ), compared with patients who did not have them (HR, 1.29; 95\% CI, 1.081.56; $P<0.006)$. After adjustment, an increase in the number of metastatic lymph nodes was associated with a decrease in overall survival in patients with up to 6 metastatic nodes (HR, 1.12; 95\% CI, 1.01-1.25; $P<0.03$ ).

The National Cancer Database contained 21,870 patients with pT3 N0 M0 or pT1-pT3 N1 M0 disease. Mortality was reduced by $29 \%$ among the 15,418 patients who were treated with radioiodine, compared with 6,452 patients who were not referred for radioiodine therapy. This advantage in favor of radioiodine therapy was found despite the remarkable fact that disease was more advanced in the patients who had radioiodine treatment: $51 \%$ of the patients had multifocal disease in the radioiodine group, versus $47 \%$ in the nonradioiodine group. Both lymph node metastases ( $74 \%$ vs. $68 \%$ ) and R1 resections (19\% vs. $15 \%$ ) were more frequent in the radioiodine group. Despite the less favorable patient population, radioiodine therapy could decrease mortality (Table 1) (22).

The statistical calculations presented by Adam et al. $(18,19)$, Orosco et al. (20), and Ruel et al. (22) were published after the ATA guidelines but should receive an adequate appraisal in a future version.

French societies, which essentially share the viewpoint of the European Association of Nuclear Medicine on radioiodine therapy, published the following recommendations: in multifocal pT1a with a total lesion size of less than $1 \mathrm{~cm}$, in pT1b N0/Nx without extrathyroidal extension, in pT1a N0/Nx with minor extrathyroidal extension, and in FTC without vascular invasion, the use of radioactive iodine is optional. In other patients with a low risk of relapse (apart from R1 and R2), including pT1b Nx/N0 with minor extrathyroidal extension, radioactive iodine treatment is recommended (23).

Patients with an intermediate risk of relapse have at least one of the following criteria, and radioiodine treatment is recommended:

- pT2 N0/Nx with minor extrathyroidal extension, or pT3a (tumor size $>4 \mathrm{~cm}$, other than NIFTP) NO/Nx with or without minor extrathyroidal extension

- pT2/pT3a N1a with limited lymph node involvement $(\leq 5$ metastatic nodes and tumor size $<2 \mathrm{~mm}$ )

- pT1a/pT1b/pT2/pT3a N1 (N1a or N1b) with involvement of intermediate to large lymph nodes, the largest metastatic node being smaller than $3 \mathrm{~cm}$

- $\mathrm{cN} 1$ disease at diagnosis (e.g., metastatic cervical lymph node confirmed by ultrasound-guided fine-needle aspiration biopsy)

- An unfavorable histologic aspect (e.g., tall cell, hobnail, or columnar cell variant of PTC; oxyphilic Hürthle cell variant of FTC)

- PTC with limited vascular invasion $(<4$ emboli) 
- In cases of radioiodine administration, positive cervical lymph nodes on posttherapy scan

\section{SPECIAL TUMOR ENTITIES}

PTMC

The British Thyroid Association (4) commented about the use of radioiodine therapy in PTMC, which is defined as a carcinoma no more than $10 \mathrm{~mm}$ in greatest dimension. PTMCs constitute approximately $30 \%$ of all DTCs and are largely responsible for the rise in the incidence of thyroid cancer seen in many countries over the last few decades. In 15 studies with 4,096 patients and a follow-up of 3.7-11.2 y, the risk for distant metastasis was $0.4 \%$. In 16 studies with 5,256 patients, there was a risk of $2.5 \%$ for locoregional recurrence and $12.3 \%-50 \%$ for locoregional lymph node involvement. Today, systematic lymph node dissection of the central compartment is no longer standard therapy, and the presence of occult lymph node metastases is therefore usually not known (cNx). The clinical outcome of PTMC is almost always very good, but there are a few exceptions: a metaanalysis that included 9,379 patients identified 32 deaths (24). Because longterm survival is nearly $100 \%$, the objective of any treatment is to reduce the small risk of locoregional recurrence and distant metastases while minimizing iatrogenic morbidity. The British Thyroid Association recommended a risk-adapted approach in PTMC. Risk factors include a tumor diameter of 6-10 mm, multifocal or bilateral tumors, an unfavorable histology with a poorly differentiated component, tumor desmoplasia or infiltrative tumor growth or incidental discovery on ${ }^{18} \mathrm{~F}-\mathrm{FDG} \mathrm{PET} / \mathrm{CT}$, and lymph node involvement at diagnosis. Nonincidental, that is, clinically detected, PTMC had an increased risk for lymph node metastases in comparison to cases discovered incidentally (25). Under these circumstances, an individual decision about radioiodine therapy can be made.

\section{NIFTP}

Diagnosing the new entity NIFTP, previously termed noninvasive follicular variant of papillary thyroid carcinoma, requires exhaustive analysis of the nodule capsule $(23,26)$, which can be difficult in large tumors and is not always included in current histologic analysis (23). In the recently published study of Thompson (7) including 94 patients with NIFTP, all patients were without evidence of disease after a median follow-up of $11.8 \mathrm{y}$. In this cohort, the diameter of the follicular neoplasms ranged from 0.5 to $9.5 \mathrm{~cm}$. All NIFTPs showed benign behavior, supporting limited surgical procedures without radioiodine ablation (7).

\section{RISK ASSESSMENT OF RADIATION EXPOSURE}

Rubino at el. reported a small risk of second malignancies for cumulated activities of more than $7.4 \mathrm{GBq}$ of ${ }^{131} \mathrm{I}$ (27). Data from the SEER Database are difficult to assess because most secondary malignancies were detected within $1 \mathrm{y}$ of radioiodine therapy, making a causal relationship unlikely. It seems more likely that these patients had access to improved diagnostic facilities, explaining a higher diagnostic yield of malignancies (28). Hirsch et al. reported on 1,943 patients, of whom $1,574(81 \%)$ were treated with radioiodine and 1,467 were followed for at least $2 \mathrm{y}$, and of these, $1,145(78 \%)$ received a cumulative dose of more than 7.4 $\mathrm{GBq}(200 \mathrm{mCi})$ of radioiodine. In patients followed for $2 \mathrm{y}$, second malignancies were diagnosed in $9 \%$ of radioiodine-treated patients and $10.5 \%$ of non-radioiodine-treated patients (29). Data from Korea on 211,360 patients analyzed the dose-dependent risk between radioiodine and leukemia (Table 2) (30). In the group treated with activities of up to $3.7 \mathrm{GBq}$, no increase in the incidence of leukemia was observed. However, there was an increased risk in the group treated with more than $3.7 \mathrm{GBq}(100 \mathrm{mCi})$, meaning that 1 in 2,000 patients treated with a radioiodine activity of more than 3.7 GBq may develop leukemia as a second malignancy. As a new aspect, the latency period before the statistically increased risk of leukemia was first observed $(\sim 8 \mathrm{mo})$ is shorter than previously assumed (30).

\section{CURRENT TREATMENT STRATEGIES}

Two important prospective randomized controlled trials have compared recombinant thyroid stimulating hormone (rhTSH) with hypothyroidism and $1.1 \mathrm{GBq}(30 \mathrm{mCi})$ versus $3.7 \mathrm{GBq}(100 \mathrm{mCi})$ of radioiodine for ablative radioiodine therapy: the ESTIMABL trial (31) and the British study "High- Versus Low-Dose Radioiodine" (HiLo trial) (32). The authors concluded that rhTSH and hypothyroidism were equally effective and that $1.1 \mathrm{GBq}$ was not inferior to $3.7 \mathrm{GBq}$. However, the trials had some drawbacks related in part to the definition of endpoints. In the ESTIMABL trial, success was defined as a stimulated thyroglobulin level of less than $1 \mathrm{ng} / \mathrm{mL}$. The authors did not report the percentages of

TABLE 2

Risk of Secondary Leukemia After Radioiodine Treatment (30)

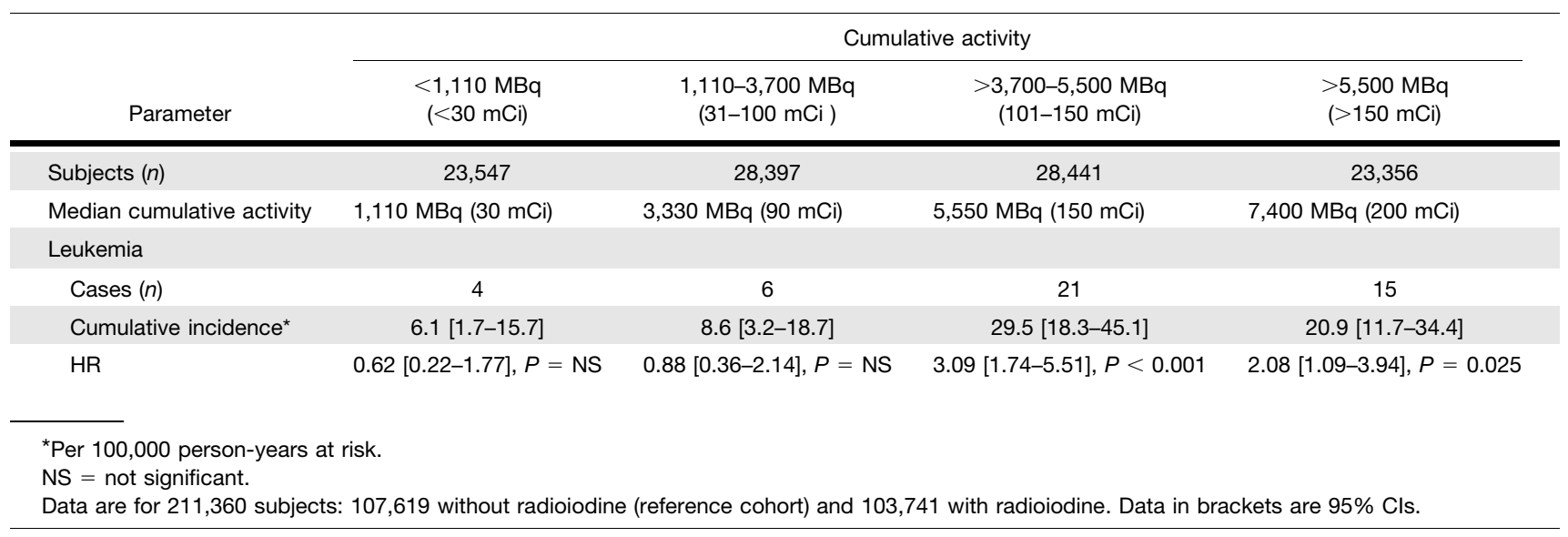


patients in whom a thyroglobulin level below the assay sensitivity was reached. A modern thyroglobulin assay with a lower functional sensitivity of $0.2 \mathrm{ng} / \mathrm{mL}$ was used. Only 24 of 684 patients underwent diagnostic whole-body scintigraphy, and neck uptake as high as $0.5 \%$ was accepted as success. In the HiLo trial, $1.1 \mathrm{GBq}$ was $5.9 \%$ less effective than $3.7 \mathrm{GBq}$ in the rhTSH group, and a second ablation was necessary in $9.5 \%$ of the $1.1-\mathrm{GBq}$ group versus $4.1 \%$ in the $3.7-\mathrm{GBq}$ group, indicating initial ablation failure. A stimulated thyroglobulin level of less than $2.0 \mathrm{ng} / \mathrm{mL}$ and thyroid uptake still as high as $0.1 \%$ were considered to indicate successful ablation. However, 68 of 438 patients had incomplete laboratory tests. Thus, the HiLo trial showed inferior results for the low-risk group (32).

The success of radioiodine therapy is critically dependent on the surgeon's experience. The more total the thyroidectomy is and the lower the remnant volume, the lower is the activity of radioiodine required for successful therapy. The clinical aim is to treat patients with the lowest single dose of radioiodine activity necessary to achieve cure. However, this is a difficult aim and the above-mentioned trials used a surrogate parameter as endpoints (i.e., stimulated thyroglobulin [rhTSH]) 8 mo after radioiodine therapy. Patients treated in Poland (33), Turkey (34), and Iran (35) needed increased rates of second ablative therapies when lower initial activities of radioiodine were used. In all 3 studies, the cumulative activities of 2 ablative therapies were higher than the radioiodine dose in the standard arm (3.7 GBq of $\left.{ }^{131} \mathrm{I}\right)$. To answer questions on disease-specific and overall survival, long-term follow-up is necessary. In our institution, low-risk patients (pT1-pT2 cN0) are treated with $2.0 \mathrm{GBq}$ of ${ }^{131} \mathrm{I}$ if larger thyroid remnants do not exist before the radioiodine ablation. The concept of a 2-GBq dose of ${ }^{131}$ I was recently confirmed by retrospective data from Munich: Todica et al. demonstrated an equally high success rate with 2 GBq $(90 \%, n=135)$ versus $3.7 \mathrm{GBq}(91 \%, n=137)$ using a thyroglobulin threshold of $0.5 \mathrm{ng} / \mathrm{mL}(36)$.

\section{Hypothyroidism Versus rhTSH}

To achieve sufficient radioiodine uptake, either hypothyroidism or rhTSH is required. rhTSH is indicated for pretherapeutic stimulation in combination with a range of 1.1-3.7 GBq (30$100 \mathrm{mCi}$ ) of radioiodine for ablation of thyroid tissue remnants in patients who have undergone a near-total or total thyroidectomy for DTC and do not have evidence of distant metastatic thyroid cancer (37). Hypothyroidism may decrease renal clearance, which is unaffected when rhTSH is used. In a study from Hänscheid et al., rhTSH reduced whole-body exposure by $35 \%$. Within the thyroid remnant, uptake after $48 \mathrm{~h}$ and residence time were not different. The specific absorbed dose to the blood was significantly $(P<0.0001)$ lower after administration of rhTSH (mean, $0.109 \pm$ $0.028 \mathrm{mGy} / \mathrm{MBq}$; maximum, $0.18 \mathrm{mGy} / \mathrm{MBq}$ ) than after thyroid hormone withdrawal (mean, $0.167 \pm 0.061 \mathrm{mGy} / \mathrm{MBq}$; maximum, $0.35 \mathrm{mGy} / \mathrm{MBq}$ ) (38).

\section{Ongoing Studies}

Because there were no prospective randomized trials comparing ablative radioiodine therapy with relinquishment of radioiodine, the following studies have been initiated:

ESTIMABL2 Trial. In the ESTIMABL2 trial (ClinicalTrials.gov identifier NCT01837745) (10,11), patients with PTC, FTC, or Hürthle cell carcinoma can be included if in stage pT1am N0 or $\mathrm{Nx}$ M0 with a sum of lesion size greater than $1 \mathrm{~cm}$ but not more than $2 \mathrm{~cm}$, or in stage pT1b N0 or Nx M0 (2010 TNM classification). Patients are randomized to receive $1.1 \mathrm{GBq}$ (30 $\mathrm{mCi}$ ) of radioiodine therapy or no radioiodine therapy. Adverse events after 3 and $5 \mathrm{y}$ are the primary endpoints.

IoN Trial. In the IoN trial (ClinicalTrials.gov identifier NCT01398085) (12), patients with PTC can be included if in stage pT1am, pT1b, pT2, or pT3 (intrathyroidal) pN0 or pN1a, and patients with minimally invasive FTC or Hürthle cell carcinoma can be included if in stage pT1b or pT2 without angioinvasion (32). Patients are randomized to receive $1.1 \mathrm{GBq}$ of ${ }^{131} \mathrm{I}$ or no radioiodine. After 6-9 mo, diagnostic radioiodine whole-body scintigraphy is planned. rhTSH-suppressive levothyroxine therapy is performed. The endpoint is progression-free survival after $5 \mathrm{y}$.

CLERAD-PROBE Trial. In the CLERAD-PROBE trial (ClinicalTrials.gov identifier NCT01704586) (13), patients with DTC (including Hürthle cell carcinoma) can be included if in stage T1b-T4 N0-N1 M0-M1 and are randomized to the competing guideline groups. In the ${ }^{124} \mathrm{I}$ arm, patients are treated following the ATA guideline risk stratification (no ${ }^{131} \mathrm{I}$ ablation in the lowrisk group; ${ }^{131} \mathrm{I}$ ablation in the high-risk group or if uptake on ${ }^{124}$ I-PET is seen outside the thyroid bed). In the standard arm, ${ }^{131}$ I ablation is performed according to the European Association of Nuclear Medicine guidelines. The primary effectiveness endpoint is mean blood dose after complete remission or after 18 mo. Secondary endpoints are quality of life (radiation side effects), tumor recurrence or progression after 3 and $10 \mathrm{y}$, and the prognostic value of thyroglobulin.

There are foreseeable problems with these 3 trials: the duration of follow-up may be too short to detect significant differences. Sawka et al. found an improved recurrence-free and overall survival only in studies with a follow-up of $10 \mathrm{y}$ or more $(8,9)$. Life expectancy is an important aspect in the decision on whether to advise the use of radioiodine therapy, especially in the younger patients-having a life expectancy of decades-in whom most cases of DTC are detected today. An open issue is survival when low activities of radioiodine are used for treatment. Verburg et al. showed that the use of more than $2 \mathrm{GBq}$ of ${ }^{131} \mathrm{I}$ resulted in an improved survival in the low- and high-risk groups, in comparison to the use of lower activities ( $\leq 2 \mathrm{GBq}$ ) (39). Thus, it is uncertain whether the aim of reducing morbidity and mortality can be realized when low activities of radioiodine are used.

\section{TREATMENT RECOMMENDATIONS}

Recommendations on whether to advise the use of radioiodine therapy have changed over time and vary among countries. The ATA guidelines on the use of radioiodine in the United States became increasingly restricted, whereas other countries are more open to recommending radioiodine therapy. Prospective randomized trials are lacking. In addition, because most of the tumors are slow-growing, studies with several thousands of patients and decades of follow-up would be needed (40). In the United States, about $50 \%$ of patient in stage I (pT1a-pT1b N0 M0 in patients $>$ 45 y old and pT1-pT3 N0-N1 M0 in patients $<45$ y old) receive radioiodine treatment. The most recent ATA guidelines did not include the latest and largest studies $(18-20,22)$. Data from the National Cancer Database showed a reduced risk for the endpoint mortality even for DTC tumors between 1 and $2 \mathrm{~cm}$ in size. Radioiodine treatment has been found to be more important for prognosis than is the extent of resection $(18,19)$. Other strategies, such as delayed radioiodine treatment in cases of increasing thyroglobulin level, have not found clinical acceptance yet. In intermediate-risk 
patients, postoperative monitoring of thyroglobulin levels alone was found to be insufficient for detection of patients with metastases $(41,42)$. Without adjuvant radioiodine therapy, thyroglobulin production by a thyroid remnant will mask early detection of recurrent disease-a particular problem because of the decreasing use of central lymph node dissection. Microscopic nodal disease will no longer be detected or treated. For intermediate-risk patients, a significant survival advantage was found for the radioiodine treatment group (22). In multifocal PTMC, the risk for recurrence was estimated to be $4 \%-6 \%$ by the ATA. Shattuck et al. analyzed the patterns of X-chromosome inactivation of multiple distinct foci of well-differentiated multifocal PTC and showed that individual tumor foci in patients with multifocal PTC often arise as independent tumors (43). Thus, in viable thyroid remnants there may be an increased risk of the de novo appearance of tumor cells, which would be controlled by radioiodine treatment. Risks and side effects of radioiodine treatment exist but are neither frequent nor of impressive clinical severity. Radiation-induced inflammation of the thyroid is among the more frequent side effects and is usually easy to treat by application of cooling ice packs or nonsteroidal antiinflammatory drugs. The Korean data indicated that leukemia occurred as a side effect only when treatment activities were above $3.7 \mathrm{GBq}$, and the likelihood was only 1 in about 2,000 therapies. In low-risk patients, adjuvant radioiodine therapy is given once with $2.0 \mathrm{GBq}$ of ${ }^{131} \mathrm{I}$. Response assessment 6-9 mo after radioiodine therapy by whole-body scintigraphy using very low activities and stimulated thyroglobulin have shown results equal to those from 3.7 GBq. Adjuvant radioiodine therapy and response assessment providing important information about the future risk of disease-specific survival should aim to routinely obviate a second ablation. Because of the difficulties with setting up prospective studies with decades of follow-up, it would be highly desirable to have a cancer registry at, minimally, a national or European level (30).

\section{CONCLUSION}

In our view, the 2015 ATA guidelines are too reluctant to recommend radioiodine treatment. Retrospective studies with large cohorts showed that patients with 1- to 2-cm PTC, and patients at intermediate risk, benefited from adjuvant radioiodine therapy regarding hard endpoints such as recurrence-free and overall survival. Radioiodine therapy is highly effective for micrometastases, which are present in a considerable number of patients, even those with low-risk tumors. DTC is a special tumor entity because of its usually slow growth pattern, often requiring many years before micrometastases have grown to clinically detectable disease. To prospectively demonstrate a benefit in hard end-outcome parameters, several thousands of patients and at least a decade of follow-up would be required. It appears questionable whether such studies will ever be performed. At present, recent studies not included in most guidelines justify the use of radioiodine not only in high-risk patients but also in those at low or intermediate risk $(18,20,22)$.

\section{REFERENCES}

1. Hertz BE, Schuller KE. Saul Hertz (1905-1950): a pioneer in the use of radioactive iodine. Endocr Pract. 2010;16:713-715.

2. Ito Y, Miyauchi A. Lateral and mediastinal lymph node dissection in differentiated thyroid carcinoma: indications, benefits, and risks. World J Surg. 2007;31:905-915.

3. Pereira JA, Jimeno J, Miquel J, et al. Nodal yield, morbidity, and recurrence after central neck dissection for papillary thyroid carcinoma. Surgery. 2005;138:1095-1100.
4. Perros P, Boelaert K, Colley S, et al.; British Thyroid Association. Guidelines for the management of thyroid cancer. Clin Endocrinol (Oxf). 2014;81(suppl 1):1122.

5. Dralle H, Musholt TJ, Schabram J, et al. German Association of Endocrine Surgeons practice guideline for the surgical management of malignant thyroid tumors. Langenbecks Arch Surg. 2013;398:347-375.

6. Haugen BR, Alexander EK, Bible KC, et al. 2015 American Thyroid Association management guidelines for adult patients with thyroid nodules and differentiated thyroid cancer: the American Thyroid Association guidelines task force on thyroid nodules and differentiated thyroid cancer. Thyroid. 2016;26:1-133.

7. Thompson LD. Ninety-four cases of encapsulated follicular variant of papillary thyroid carcinoma: a name change to noninvasive follicular thyroid neoplasm with papillary-like nuclear features would help to prevent overtreatment. Mod Pathol. 2016;29:698-707.

8. Sawka AM, Thephamongkhol K, Brouwers M, et al. Clinical review 170: a systematic review and metaanalysis of the effectiveness of radioactive iodine remnant ablation for well-differentiated thyroid cancer. J Clin Endocrinol Metab. 2004;89:3668-3676.

9. Sawka AM, Brierley JD, Tsang RW, et al. An updated systematic review and commentary examining the effectiveness of radioactive iodine remnant ablation in well-differentiated thyroid cancer. Endocrinol Metab Clin North Am. 2008;37: $457-480$.

10. Differentiated thyroid cancer: is there a need for radioiodine ablation in low risk patients? (ESTIMABL2). ClinicalTrials.gov website. https://clinicaltrials.gov/ ct2/show/NCT01837745. Published April 23, 2013. Updated May 16, 2017. Accessed May 11, 2018.

11. Mallick U, Harmer C, Hackshaw A, Moss L. Iodine or not (IoN) for low-risk differentiated thyroid cancer: the next UK national cancer research network randomized trial following HiLo. Clin Oncol (R Coll Radiol). 2012;24:159161.

12. IoN: is ablative radio-iodine necessary for low risk differentiated thyroid cancer patients (IoN). ClinicalTrials.gov website. https://clinicaltrials.gov/ct2/show/ NCT01398085. Published July 20, 2011. Updated October 26, 2016. Accessed May 11, 2018.

13. I-124 PET/CT based remnant radioiodine ablation decision concept in differentiated thyroid cancer (CLERAD-PROBE). ClinicalTrials.gov website. https:// clinicaltrials.gov/ct2/show/NCT01704586. Published October 11, 2012. Updated May 3, 2017. accessed May 11, 2018.

14. Carling T, Carty SE, Ciarleglio MM, et al American Thyroid Association Surgical Affairs Committee. American Thyroid Association design and feasibility of a prospective randomized controlled trial of prophylactic central lymph node dissection for papillary thyroid carcinoma. Thyroid. 2012;22:237-244.

15. Haugen BR. 2015 American Thyroid Association management guidelines for adult patients with thyroid nodules and differentiated thyroid cancer: what is new and what has changed? Cancer. 2017;123:372-381.

16. Verburg FA, Aktolun C, Chiti A, et al.; EANM and the EANM Thyroid Committee. Why the European Association of Nuclear Medicine has declined to endorse the 2015 American Thyroid Association management guidelines for adult patients with thyroid nodules and differentiated thyroid cancer. Eur J Nucl Med Mol Imaging. 2016;43:1001-1005.

17. Dietlein M, Eschner W, Grünwald F, et al. Procedure guidelines for radioiodine therapy of differentiated thyroid cancer: version 4 [in German]. Nucl Med (Stuttg). 2016;55:77-89.

18. Adam MA, Pura J, Gu L, et al. Extent of surgery for papillary thyroid cancer is not associated with survival: an analysis of 61,775 patients. Ann Surg. 2014;260: 601-605.

19. Adam MA, Pura J, Goffredo P, et al. Presence and number of lymph node metastases are associated with compromised survival for patients younger than age 45 years with papillary thyroid cancer. J Clin Oncol. 2015;33:2370-2375.

20. Orosco RK, Hussain T, Brumund KT, Oh DK, Chang DC, Bouvet M. Analysis of age and disease status as predictors of thyroid cancer-specific mortality using the Surveillance, Epidemiology, and End Results database. Thyroid. 2015;25:125132.

21. Chow SM, Yau S, Kwan CK, et al. Local and regional control in patients with PTC: specific indications of external radiotherapy and radioactive iodine. Endocr Relat Cancer. 2006;13:1159-1172.

22. Ruel E, Thomas S, Dinan M, et al. Adjuvant radioactive iodine therapy is associated with improved survival for patients with intermediate-risk papillary thyroid cancer. J Clin Endocrinol Metab. 2015;100:1529-1536.

23. Zerdoud S, Giraudet AL, Leboulleux S, et al. Radioactive iodine therapy, molecular imaging and serum biomarkers for differentiated thyroid cancer: 2017 guidelines of the French Societies of Nuclear Medicine, Endocrinology, Pathology, Biology, Endocrine Surgery and Head and Neck Surgery. Ann Endocrinol (Paris). 2017;78:162-175. 
24. Roti E, Degli Uberti EC, Bondanelli M, et al. Thyroid papillary microcarcinoma: a descriptive and meta-analysis study. Eur J Endocrinol. 2008;159:659-673.

25. Mehanna H, Al-Maqbili T, Carter B, et al. Differences in the recurrence and mortality outcomes rates of incidental and nonincidental papillary thyroid microcarcinoma: a systematic review and meta-analysis of 21329 person-years of follow-up. J Clin Endocrinol Metab. 2014;99:2834-2843.

26. Xu B, Tallini G, Scognamiglio T, et al. Outcome of large noninvasive follicular thyroid neoplasm with papillary-like nuclear features. Thyroid. 2017;27:512517.

27. Rubino C, de Vathaire F, Dottorini ME, et al. Second primary malignancy in thyroid cancer patients. Br J Cancer. 2003;89:1638-1644.

28. Brown AP, Chen J, Hitchcock YJ, et al. The risk of second primary malignancies up to three decades after treatment of differentiated thyroid cancer. J Clin Endocrinol Metab. 2008;93:504-515.

29. Hirsch D, Shohat T, Gorshtein A, et al. Incidence of nonthyroidal primary malignancy and the association with ${ }^{131} \mathrm{I}$ treatment in patients with differentiated thyroid cancer. Thyroid. 2016;26:1110-1116.

30. Seo GH, Cho YY, Chung JH, et al. Increased risk of leukemia after radioactive iodine therapy in patients with thyroid cancer: a nationwide, population-based study in Korea. Thyroid. 2015;25:927-934.

31. Schlumberger M, Catargi B, Borget I, et al. Strategies of radioiodine ablation in patients with low-risk thyroid cancer. N Engl J Med. 2012;366:1663-1673.

32. Mallick U, Harmer C, Yap B, et al. Ablation with low-dose radioiodine and thyrotropin alfa in thyroid cancer. N Engl J Med. 2012;366:1674-1685.

33. Kukulska A, Krajewska J, Gawkowska-Suwinska M, et al. Radioiodine thyroid remnant ablation in patients with differentiated thyroid carcinoma (DTC): prospective comparison of long-term outcomes of treatment with 30, 60 and 100 mCi. Thyroid Res. 2010;3:9.

34. Caglar M, Bozkurt FM, Akca CK, et al. Comparison of 800 and $3700 \mathrm{MBq}$ iodine-131 for the postoperative ablation of thyroid remnant in patients with lowrisk differentiated thyroid cancer. Nucl Med Commun. 2012;33:268-274.

35. Fallahi B, Beiki D, Takavar A, et al. Low versus high radioiodine dose in postoperative ablation of residual thyroid tissue in patients with differentiated thyroid carcinoma: a large randomized clinical trial. Nucl Med Commun. 2012;33:275282.

36. Todica A, Haidvogl S, Fendler WP, et al. Effectiveness of reduced radioiodine activity for thyroid remnant ablation after total thyroidectomy in patients with low to intermediate risk differentiated thyroid carcinoma. Nuklearmedizin. 2017;56: 211-218.

37. Thyrogen: thyrotropin alfa. European Medicines Agency website. http://www. ema.europa.eu/ema/index.jsp?curl=pages/medicines/human/medicines/000220/ human_med_001094.jsp\&mid=WC0b01ac058001d124. Published February 13, 2008. Updated January 11, 2013. Accessed May 11, 2018.

38. Hänscheid H, Lassmann M, Luster M, et al. Iodine biokinetics and dosimetry in radioiodine therapy of thyroid cancer: procedures and results of a prospective international controlled study of ablation after rhTSH or hormone withdrawal. J Nucl Med. 2006;47:648-654.

39. Verburg FA, Mäder U, Reiners C, et al. Long-term survival in DTC is worse after low-activity initial post-surgical ${ }^{131} \mathrm{I}$ therapy in both high- and low-risk patients. J Clin Endocrinol Metab. 2014;99:4487-4496.

40. Dietlein M, Verburg FA, Luster M, et al. One should not just read what one believes: the nearly irresolvable issue of producing truly objective, evidencebased guidelines for the management of differentiated thyroid cancer. Eur J Nucl Med Mol Imaging. 2011;38:793-798.

41. Park EK, Chung JK, Lim IH, et al. Recurrent/metastatic thyroid carcinomas false negative for serum thyroglobulin but positive by posttherapy I-131 whole body scans. Eur J Nucl Med Mol Imaging. 2009;36:172-179.

42. Robenshtok E, Grewal RK, Fish S, et al. A low risk postoperative nonstimulated serum thyroglobulin level does not exclude the presence of radioactive iodine avid metastatic foci in intermediate-risk differentiated thyroid cancer patients. Thyroid. 2013;23:436-442.

43. Shattuck TM, Westra WH, Ladenson PW, et al. Independent clonal origins of distinct tumor foci in multifocal papillary thyroid carcinoma. $N$ Engl $\mathrm{J}$ Med. 2005;352:2406-2412. 